\title{
Small-Scale Planktonic Structure: Persistence AND TROPHIC CONSEQUENCES
}

\author{
By Timothy J. Cowles, Russell A. Desiderio \\ and Mary-Elena Carr
}

$\mathrm{M}$ ods limit our ability to resolve planktonic distributions over vertical scales less than a few meters. Estimates of in situ biological rates also are limited by equipment and sampling resolution. It has been obvious to plankton researchers for decades that the uncertainties created by sampling limitations are complicated further by vertical and horizontal variability in plankton distributions (often called patchiness). Although patches of phytoplankton (usually undetected) were recognized as essential for zooplankton growth and survival (e.g., Mullin and Brooks, 1976; Dagg, 1977), the distribution, size, and concentration of phytoplankton patches have been viewed as random or stochastic (e.g., Fasham, 1978). If phytoplankton patchiness is characterized by randomness, the contribution of this variability to our estimates of phytoplankton biomass may be removed by averaging over larger scales, and samples obtained with conventional sampling equipment can be used with some confidence. On the other hand, if patchiness is nonrandom on spatial or temporal scales that are difficult to resolve with conventional methods, then conventional sampling may alias estimates of planktonic distributions and rate processes such as grazing and growth.

It is generally thought that biological structure is linked to physical processes (Denman and Powell, 1984; Mackas et al., 1985; Owen. 1989) including surface waves (and wave breaking), internal waves, Langmuir circulation, horizontal

Timothy J. Cowles and Russell A. Desiderio, College of Oceanic and Atmospheric Sciences. 104 Ocean Admin Bldg., Oregon State University. Corvallis. OR 97331-5503., USA. Mary-Elena Carr, Jet Propulsion Laboratory, 4800 Oak Grove Dr., Pasadena. CA 91101-8099. USA. intrusions, shear instabilities, convective overturns, and salt-fingering, all of which can lead to localized intermittent vertical mixing and redistribution of biomass (Denman and Gargett, 1983; Denman and Powell, 1984: Weller and Price, 1988; Owen, 1989). Turbulence and other small-scale physical processes are proposed to constrain a wide range of biological processes, such as nutrient uptake by phytoplankton (Goldman, 1988), photosynthesis in fluctuating light fields (Marra. 1978), grazing on phytoplankton by zooplankton (Mullin and Brooks, 1976: Cowles et al., 1988; Rothschild and Osborne, 1988), larval fish survivorship (Lasker, 1975), and the vertical distribution patterns of planktonic organisms, from bacteria to larval fish (Mitchell and Fuhrman. 1989: Owen. 1989: Denman and Gargett, 1988; Lasker, 1975; Bjornsen and Nielsen. 1991).

Observations of small-scale $(<1 \mathrm{~m})$ vertical variability in biological structure in the upper ocean began with early in situ observations of Bainbridge (1952) and the high-resolution collections of Cassie (1963) and have continued with recent centimeter-scale collections of Owen (1989), Mitchell and Fuhrman (1989). Bjornsen and Nielsen (1991). and Donaghay et al., (1992). However, with the exception of some recent work (Cowles et al.. 1990; Donaghay et al., 1992: Cowles and Desiderio, 1993) previous small-scale biological observations have lacked concurrent measurements of physical variables on the appropriate time and space scales. These recent observations confirm that any advances in understanding the response of small-scale biological structure to physical forcing require coincident measurements of physical and biological parameters over the same range of time and space scales.
In the course of developing a microstructure fluorescence sensor (Cowles et al., 1990; Cowles and Desiderio, 1993; Desiderio et al., 1993), we observed small-scale vertical structure of phytoplankton biomass in conjunction with fine-scale and microscale physical structure. We found that thin layers of locally enhanced phytoplankton biomass are common over the continental shelf and at open ocean locations, and that these layers are usually between 10 and $50 \mathrm{~cm}$ thick. As one might expect, some of these thin layers of pigment fluorescence are associated with temperature steps and/or local minima in turbulence. More surprising. however, was the finding that some individual thin layers persist as local maxima for periods of hours. Subsequent observations by our group and by others (e.g.. Donaghay et al., 1992) have confirmed that these sub-1-m scale local maxima can be detected across a range of coastal and oceanic locations when the appropriate high-resolution instrumentation is used.

These recent observations suggest that estimates of biological rate processes obtained by coarser-scale conventional sampling (e.g., $\sim 5-10-\mathrm{m}$ bottle spacing) may have missed the contribution of persistent sub-1-m scale structure. The common occurrence and persistence of these features suggest that it is necessary to quantify the contribution of thin layers to biological rate processes in the upper ocean. Relative to the average background concentration, thin layers of enhanced biomass may be characterized by high autotrophic growth rates, increased ingestion, growth, and reproduction by $\mathrm{mi}$ croheterotrophs, microzooplankton. and mesozooplankton, locally higher nutrient and particulate flux, and higher nutrient regeneration rates. It is likely that locally 
higher rates on these small-scale structures make a previously undetected contribution to carbon flux across a range of temporal and spatial scales.

In this paper we provide evidence of the existence and duration of layers in the open ocean (see Temporal Persistence of Small-Scale Features), show optical differences between nearby layers that imply persistence (see In Situ Characterization of Small-Scale Features), suggest a framework in which to understand the persistence or erasure of layers (see How Might Small-Scale Planktonic Features Persist?), and finally provide an assessment of the possible trophic impact of persistent layers (see Trophic Consequences of Persistent Small-Scale Structure).

\section{Temporal Persistence of Small-Scale Features}

We were intrigued to find small-scale planktonic features that persisted for hours. It seemed unlikely that physical processes would be quiescent enough to permit such persistence, and estimates of persistence times based on canonical diffusivities yield time scales of minutes. not hours. Our time-series observations suggest that $10-50-\mathrm{cm}$ scale features persist over ecologically relevant time scales for planktonic organisms. Over 170 layers of particle scattering were observed to persist for at least 4-6 $\mathrm{h}$ in an open ocean site off the California coast (Carr et al.. unpublished data).

Observations with a profiling microstructure fluorometer (Cowles et al., 1990; Cowles et al., 1993: Desiderio et al., 1993) demonstrated that the vertical patterns of phytoplankton pigment fluorescence often have local maxima within narrow layers $(10-50 \mathrm{~cm}$ in thickness). In addition, some of these narrow local maxima were present at the onset of a time series and were still present when sampling terminated. We illustrate this phenomenon with a time series of profiles collected 100 miles off the Oregon coast. These profiles, taken $\sim 12$ min apart, revealed a variable physical structure (Fig. 1 , a and b) and considerable small-scale variability in phytoplankton structure, based on the measured fluorescence (Fig. Ic: in these figures each succeeding profile is offset to show each trace more clearly). The profiles indicate internal wave displacement of $\sim 2.2 \mathrm{~m}$ during the time series, which is consistent with other observations of internal wave activity for
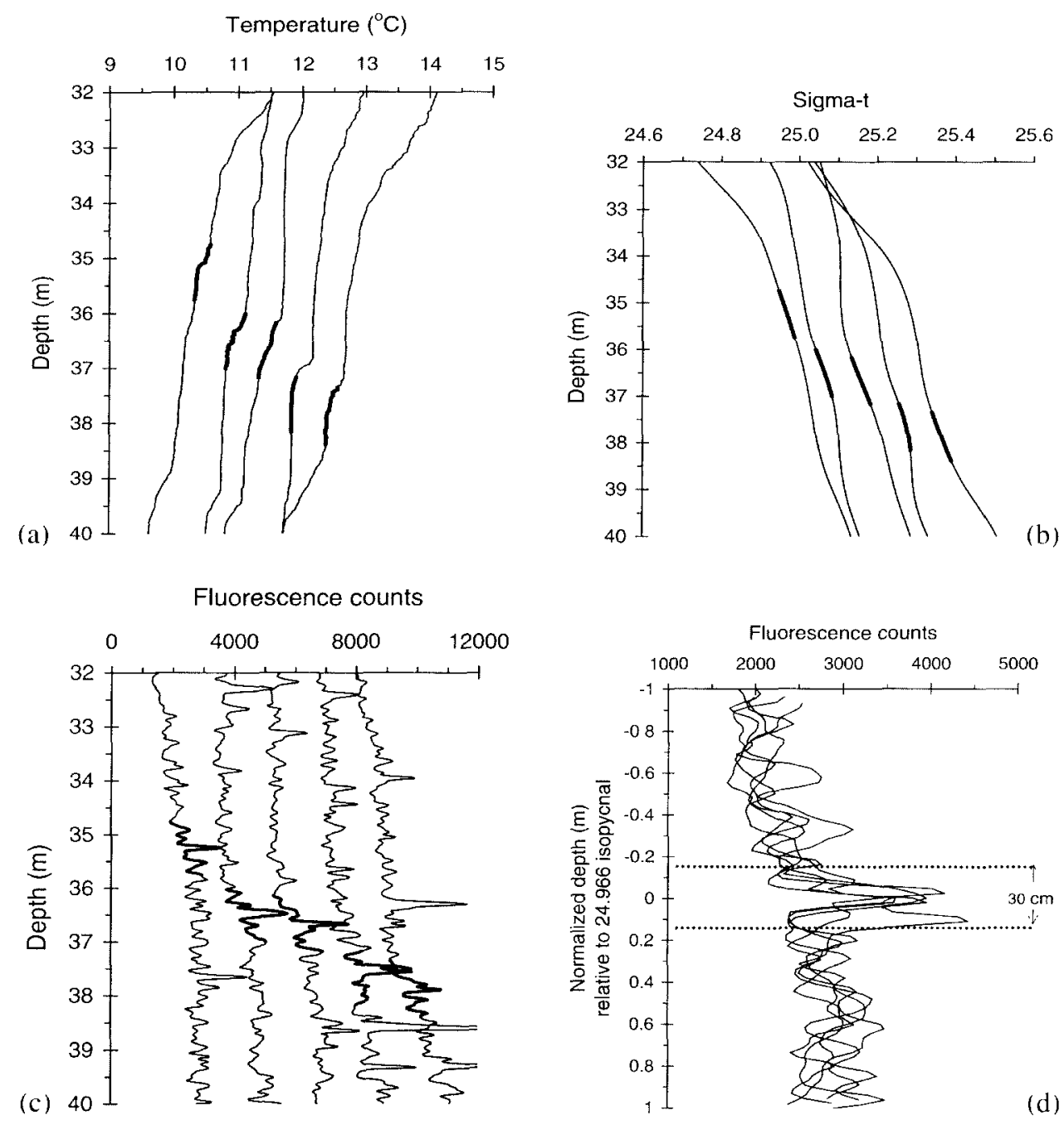

Fig. I: Vertical detail $(32-40 \mathrm{~m})$ of (a) temperature. (b) sigma-t, and (c) fluorescence from five profiles spanning approximately $1 \mathrm{~h}$ during a cruise off the Oregon coast. The profiles in $\boldsymbol{a}, \boldsymbol{b}$, and $\boldsymbol{c}$ are offset to show each trace. In $\boldsymbol{a}, \boldsymbol{b}$, and $\boldsymbol{c}$ the $1-\mathrm{m}$ segments of each profile that were centered on an isopycnal of 24.966 sigma-t units are highlighted. In $\boldsymbol{d}$, those 1-m vertical segments of fluorescence identified in $c$ are plotted relative to the depth of the 24.966 isopycnal $( \pm 0.003$ sigma-t units). The local maximum in fluorescence now is seen as a persistent thin laver.

this region. Some of the small-scale fluorescence maxima were associated with specific temperature or density intervals. The profiles shown in Figure 1 have a persistent phytoplankton fluorescence feature centered within 0.003 sigma-t units of the 24.966 isopycnal (Fig. 1d). The measured fluorescence of that narrow feature did not vary by more than $10 \%$ during the time-series.

Additional evidence for persistent features comes from Donaghay et al. (1992), who reported significant enhancements in nutrients, primary production, and meroplankton larval abundance within a thin layer in a stratified embayment. In addition. Oldham and Imberger (1992) reported 20-cm-thick layers of oxygen mi- crostructure that persisted for hours in Australian lakes. In this issue, papers by Holliday et al. and Hanson and Donaghay provide additional examples of persistent small-scale features.

It is important to note that these observations of persistent small-scale structure have been made possible through advances in instrumentation that permit sampling of the upper ocean over a different range of time and space scales than could be accomplished even a decade ago. In situ optical instruments are particularly well-suited to reveal small-scale biological structure because these devices have high sampling rates and are compact enough to include on profiling systems. 


\section{In Situ Characterization of Small-Scale Features}

We have approached the detection and characterization of persistent small-scale structure through the use of free-fall profiling systems that can carry CTDs and the new generation of biooptical instrumentation. These physical and optical tools permit us to describe individual small-scale features and to distinguish differences between nearby features. For example, while profiling the upper $100 \mathrm{~m}$ of the water column off the Oregon coast with a fiber-optic microstructure fluorometer (see Desiderio et al., 1993), we observed spectral changes in the in situ fluorescence emission due to phycobilin pigments. These shifts of the emission peaks in the fluorescence spectra suggested a taxonomic change from cyanobacteria to cryptomonads within the thermocline that was confirmed with discrete sample collections (Cowles et al., 1993).

Our present free-fall instrumentation package was developed in conjunction with Dr. J. Ronald Zaneveld of Oregon State University and has a Sea-Bird $911+$ CTD, two multiwavelength absorption meters (WetLabs ac-9), and a multiexcitation spectrofluorometer (WetLabs SAFIRE) as its basic configuration. Additional instruments can be added as needed. We deploy the package on a loose data tether, with sufficient buoyancy on the package to obtain descent rates of $15-20 \mathrm{~cm} / \mathrm{s}$. These slow descent rates allow data acquisition on centimeter spatial scales and result in multiparameter characterization of small-scale features (see Hanson and Donaghay, 1998, for additional examples).

We have obtained many profiles that reveal distinct shifts in spectral absorption and spectral fluorescence properties between nearby small-scale features. For example, a series of vertical profiles from East Sound, WA, revealed several distinct layers of phytoplankton biomass (Fig. 2a). Simultaneous observations of the absorption spectra and fluorescence emission spectra indicate that the layers marked B and D in Figure 2a had different optical characteristics (the amplitudes of the signals were scaled to show spectral differences; see figure legend for details). Layer B (at $16.7 \mathrm{~m}$ ) exhibited relatively more absorption in the violet and blue and less in the green to orange region of the spectrum than layer D (Fig. $2 b)$, which in turn showed relatively more fluorescence emission at $450-550$ $\mathrm{nm}$ when excited by $228-\mathrm{nm}$ light (Fig. 2c). This suggests a different community composition or photoadaptive state even within close spatial proximity, providing indirect evidence that these layers have persisted long enough for their different optical characteristics to have developed independently.

These data, and those of other investigators working with small-scale structure, indicate that the in situ observational tools are now available to fingerprint adjacent small-scale features and evaluate their differences based on physical/optical characteristics. New water sampling approaches are also being applied to assist in these comparisons and will permit rate process experiments to be conducted.

\section{How Might Small-Scale Planktonic Features Persist?}

The relative importance of small-scale planktonic layers to trophic processes will depend on the number of persistent layers and how long they persist. Both are dependent on physical and biological processes. The formation of layers, for example, could result from physical processes, such as 1) water column stratification due to solar warming, horizontal intrusions, etc., that create density gradients that might collect sinking particles (e.g., Lande and Wood, 1987; MacIntyre et al., 1995), 2) locally "quiet" vertical intervals that have lower than average turbulent mixing such that local phytoplankton accumulations are not dispersed, 3 ) interac-
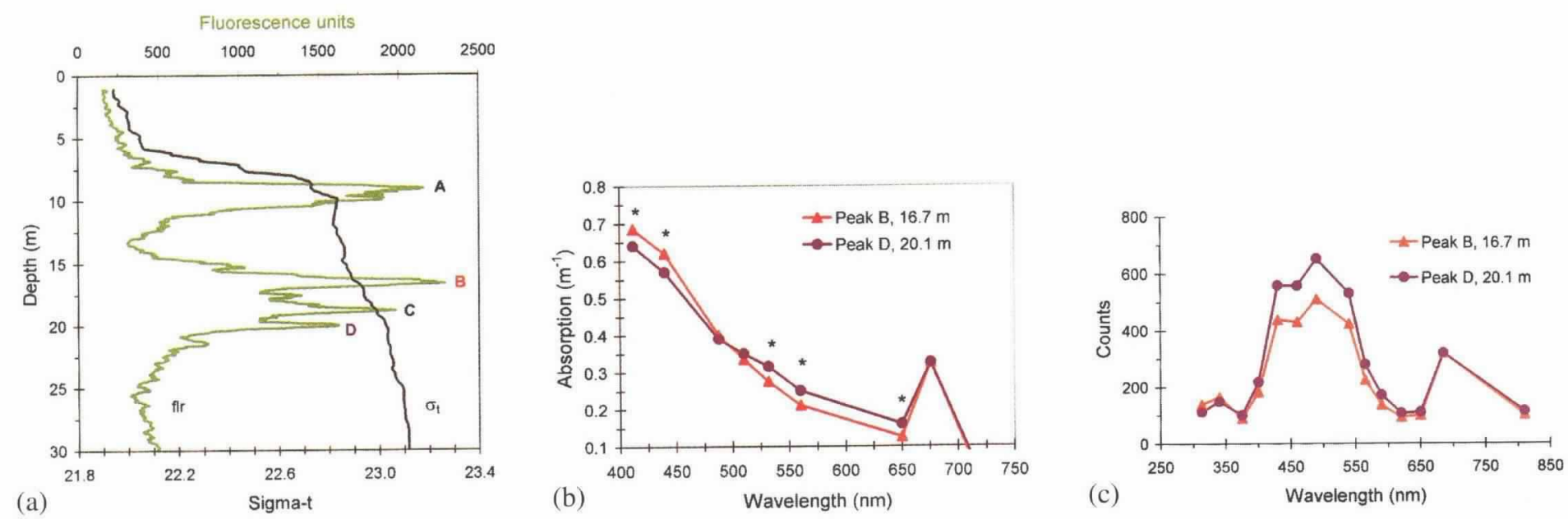

Fig. 2: (a) Characteristic profile of phytoplankton pigment fluorescence and sigma-t during a series of profiles in East Sound, WA, in the summer of 1995. Chlorophyll concentration at $10 \mathrm{~m}$ depth was $\sim 5 \mu \mathrm{g} l^{-1}$. (b) Absorption spectra from peaks $B$ and $D$ in a, where spectra have been corrected for temperature and scattering. Each point is the mean of 10 measurements obtained within its layer from successive profiles with an ac-9. To illustrate the relative shapes of the absorption spectra, the absorption values for layer $D$ were scaled by multiplying them by a factor of $a 676(B) / a 676(D)$. Those points at a given wavelength marked with an asterisk are significantly different at the $95 \%$ confidence level. (c) Fluorescence emission spectra from 228-nm excitation for peaks B and D. Emission values for layer $D$ were scaled by multiplying them by a factor of emission685(B)/emission685(D). The deeper layer at $20 \mathrm{~m}$ has significantly more relative emission between 450 and $550 \mathrm{~nm}$ than does the layer near $17 \mathrm{~m}$, indicating either a relatively higher concentration of fluorescent dissolved organic material, or a relatively lower amount of absorption at the excitation wavelength by nonfluorescent moieties. 
tion of vertical gradients in horizontal velocity (shear) with local stratification that could result in the horizontal dispersion (stretching) of biological distributions. like those observed by Kullenberg (1974) by injection of dye into stratified waters, and 4) interaction of internal waves and horizontal biomass gradients as proposed by Franks (1995) in his model of thin layer formation.

Layer persistence or erasure will be dependent on the interaction between the physical processes of upper ocean mixing and the biological processes of phytoplankton growth and zooplankton grazing. The intermittent occurrence and variable intensity of mixing events (e.g.. Moum et al., 1989) will determine the time scales of layer persistence.

The mixing environment can be characterized, in part, by the interplay of the destabilizing effect of velocity shear and the stabilizing influence of the density gradient. These two parameters provide a framework to understand whether conditions are favorable for layer persistence or not. The transition from stability to mixing is usually identified by low values $(<0.25)$ of the Richardson number, $R_{\text {, }}$ (where $R_{1}=N^{2} / s^{2}$, given by the ratio of the square of the Brunt-Väisälä frequency, $\mathrm{N}^{2}$, and the squared shear, $\mathrm{s}^{2}$ ). Persistent local minima in shear and local maxima in the density gradient (represented by $\mathrm{N}^{2}$ ) create regions of stability that allow thin layers to persist. Conversely, local increases in shear and/or local decreases in the density gradient can erase existing small-scale structure through shear instabilities. It is noteworthy that the most striking examples of persistent thin layers are observed in highly stratified environments (Donaghay et al., 1992).

But biological factors, namely phytoplankton growth and zooplankton grazing, also will contribute to the persistence/erasure of thin layers. The balance between growth and mixing is crucial for layer persistence. Under conditions of relatively little mixing, phytoplankton growth could account for the maintenance of a thin layer of phytoplankton biomass.

Any combination of competing physical and biological processes may be present under the range of conditions experienced within the euphotic zone. The papers in this issue provide support for the view that we cannot adequately understand the role of persistent small-scale biological/physical structure in trophic dynamics until we have a clearer picture of the contribution of thin layers to overall rate processes, and a more quantitative description of the physical and biological phase space in which we find these features.

\section{Trophic Consequences of Persistent Small-Scale Structure}

As mentioned earlier, the relative importance of persistent structure to trophic dynamics depends on the interaction of physical mixing processes. phytoplankton growth, and zooplankton grazing/growth. Now that the tools are available to characterize and evaluate locally enhanced thin layers of nutrients, phytoplankton, and microzooplankton, we need to compare these high-resolution observations with estimates of distributions and process rates obtained with conventional sampling devices.

Conventional sampling for microzooplankton grazers occurs on vertical scales of 5-10 $\mathrm{m}$ and typically employs 10-1 Niskin bottles on a CTD/rosette system to collect discrete water samples for enumeration of microzooplankton. Each bottle spans $>0.5 \mathrm{~m}$, and the CTD/rosette system moves vertically during sampling due to ship motion. Therefore enumeration of a sample from this conventional sampling approach yields an estimated population size that is the average of the local minima and maxima of microzooplankton abundance over a $\sim 2-\mathrm{m}$ interval. In situ biomass estimates of larger zooplankton (mesozooplankton $>200 \mu \mathrm{m}$ ) are usually made over vertical intervals of at least $10-25 \mathrm{~m}$. The microzooplankton and mesozooplankton biomass profiles that result from this sampling approach, in conjunction with estimates of the vertical distribution of phytoplankton biomass, usually form the basis for estimates of water column grazing and secondary production (e.g., Dam et al., 1993).

In contrast to coarse-scale conventional sampling, fine-scale sampling by Bjornsen and Nielsen (1991) indicated that microzooplankton grazers can be much more aggregated (within 20-50-cm intervals) than could be discerned by conventional sampling. Laboratory experiments have shown that grazers can detect and stay within layers of food (e.g., Tesilius. 1992) and can discriminate between food items on the basis of chemical signals (Cowles et al., 1988). If mi- crograzers aggregate at this scale on their food supply in the field, and their food supply is distributed in narrow, persistent features, then the local grazing and growth rates of those grazers will be quite different than that estimated from coarser-scale sampling.

We have used these contrasting assessments of phytoplankton and microzooplankton biomass to compare estimates of the loss of phytoplankton to grazing within the euphotic zone, and to compare the individual and population growth rates for microzooplankton distributions defined by these different biomass profiles. We present here some simple calculations of grazer growth rates that help to illustrate the trophic consequences of persistent layers.

Microzooplankton grazing and growth can be modeled by a functional specific growth response for a generic ciliate (Fig. $3)$. This idealized growth rate function yields a doubling time of $\sim 22 \mathrm{~h}$ at saturating food conditions $\left[\mathrm{e}^{r t}=2\right.$, when the growth rate $(r)$ approaches 0.032$]$. We have used this response to estimate the specific growth rate for micrograzers as a function of food concentration. For example, if an assemblage of micrograzers

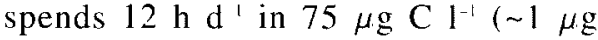
chlorophyll $\mathrm{a} \mathrm{l}^{-1}$ ) and $12 \mathrm{~h} \mathrm{~d}^{-1}$ at $15 \mu \mathrm{g} \mathrm{C}$ $1^{-1}$, its daily growth will be $>1.8$ times larger than if it spends $24 \mathrm{~h} \mathrm{~d}^{-1}$ at $15 \mu \mathrm{g}$ $\mathrm{C} \mathrm{I}^{-1}$. (This estimate assumes grazing over

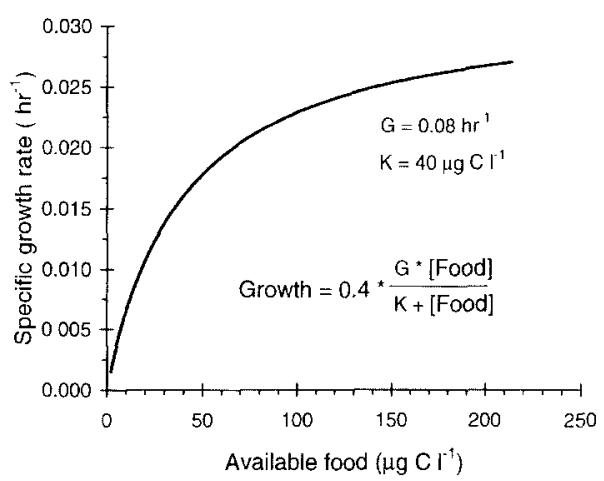

Fig. 3: Theoretical growth response of a small planktonic grazer, such as a ciliate, as a function of food availability. $K$ $\left[\mu g \mathrm{Cl}^{-1}\right]$ represents the food concentration at half saturation rate; $G\left[h^{\prime}\right]$ is the ingestion rate normalized to grazer body weight; and 0.4 represents a (typical) $40 \%$ growth efficiency (Caron and Goldman, 1990). A food concentration of 75 $\mu \mathrm{g} \mathrm{C} l^{-1}$ roughly corresponds to $I \mu \mathrm{g}$ chlorophyll a $l^{\prime}$. 
a 24-h interval, but the concept is valid for grazers that have a diel feeding cycle). This represents a substantial enhancement of growth rate for grazers that spend time within persistent layers that have locally higher food concentrations.

The consequences of estimating grazer growth rates based on coarse- versus finescale sampling can be examined by considering the following one-dimensional model. Consider a $10-\mathrm{m}$ portion of the water column that has two 0.5 -m-thick layers with a phytoplankton concentration of $75 \mu \mathrm{g} \mathrm{C} \mathrm{I}^{-1}$; the background concentration is $15 \mu \mathrm{g} \mathrm{C} 1$ '. If the thin layers of phytoplankton in this water column are not sampled (as might occur with conventional coarse-scale sampling), then the background food concentration would be used to estimate a specific growth rate of $0.009 \mathrm{~h}^{\prime}$ for the grazers present (Fig. 3). If coarse-scale sampling results in water collection only at the thin layers, then the average food concentration for this $10-\mathrm{m}$ interval will be overestimated. A 10-1 Niskin bottle sample could intersect a 50$\mathrm{cm}$ layer plus $\sim 75 \mathrm{~cm}$ on either side of the layer, resulting in a mean phytoplankton concentration of $\sim 30 \mu \mathrm{g} \mathrm{Cl}^{-1}$. This food concentration would yield an estimated specific growth rate of $0.014 \mathrm{~h}$ '. In these cases of undersampled food distributions, the estimated specific growth rates of individual grazers are independent of their vertical position due to the assumption that the food distribution is uniform.

A wider range of specific growth rates for grazers would result if the vertical distribution of thin layers of phytoplankton is resolved with fine-scale sampling: the magnitude of the mean growth rate of the grazer population then will depend on the grazer distribution. For the case in which the grazer vertical distribution is uniform, the estimated mean specific growth rate would be $0.010 \mathrm{~h}^{-1}$. However, if the grazer distribution is identical to the food distribution, then the mean growth rate would be $0.013 \mathrm{~h}^{1}, 30 \%$ more than that in the uniform grazer distribution case, and 50\% more than if the thin layers of food were not sampled. And. if all of the grazers were able to congregate on the thin layers, then the specific growth rate of the population would be $0.021 \mathrm{~h}, 110 \%$ more than the mean specific growth rate for the uniform grazer distribution case.

It is expected that the consequences of grazer aggregation on layers of enhanced food will be more important for cases in which the background food concentration is relatively low; the functional dependence of grazer growth on food availability (Fig. 3) shows that at higher food concentrations the growth rate saturates, diminishing the growth bonus realized by aggregating grazers.

The calculations presented above indicate that the trophic impact of persistent small-scale phytoplankton structure is dependent, to a large extent, on the active and/or passive accumulation of individual grazers onto local food maxima. The degree to which grazers accumulate will determine whether persistent food layers lead to much higher population growth rates than would occur in the absence of thin layers. As shown in the paper by Holliday et al. (1998), new acoustic methods are now able to provide resolution of zooplankton distribution over the relevant vertical scales and will provide essential evidence for the trophic linkages between phytoplankton and zooplankton small-scale structure.

\section{Summary}

Small-scale physical, biological, and chemical processes are the dominant, and most immediately relevant, processes for planktonic organisms. The existence of persistent thin layers suggests that patchiness in vertical structure is more than ephemeral random structure, and may represent undersampled, persistent, sub-1-m scale structure. Observations of persistent fluorescence microstructure suggest that the trophic dynamics and biological rate processes of the upper water column are constrained by both fine-scale and microscale physical processes, such that local maxima in biological rate processes occur within thin layers or sheets. It is likely that conventional sampling may result in aliased resolution of these features, as well as aliased estimates of the associated rate processes, and may lead to erroneous estimates of oceanic carbon flux. The frequent occurrence of thin layers and the temporal/spatial coherence of these layers suggest that we should evaluate their contribution to upper ocean biological processes, and determine the correlation between these small-scale structures and physical processes.

\section{Acknowledgements}

The work described in this article has been supported by the Office of Naval Research and the National Science
Foundation. We are indebted to Dr. James Moum for his collaboration in the integration of a fiber-optic fluorescence sensor on his microstructure profiling system.

\section{References}

Bainbridge, R., 1952: Underwater observations on the swimming of marine zooplankton. $J$. Mar. Biol. Assoc. UK, 31, 107-112.

Bjornsen, P.K. and T.G. Nielsen, 1991: Decimeter scale heterogeneity in the plankton during pycnocline bloom of Gyrodinium aureolum. Mar. Ecol. Prog. Ser., 73, 263-267.

Caron, D.A. and J.C. Goldman, 1990: Protozoan nutrient regeneration. In: Ecology of Marine Protozoa. G.M. Capriulo, ed. Oxford Univ. Press, New York. 283-306.

Cassie, R.M., 1963: Microdistribution of plankton. Oceanogr. Mar. Biol. Ann. Rev.. 1, 223-252.

Cowles, T.J. and R.A. Desiderio, 1993: Resolution of biological microstructure through in situ fluorescence emission spectra. Oceanography, 6, 105-111.

R.A. Desiderio, J.N. Moum, M.L. Myrick. D. Garvis and S.M. Angel, 1990: Fluorescence microstructure using a laser/fiber optic profiler. Ocean Optics X, Proc. SPIE, 1302, 336-345.

_- - . R.A. Desiderio and S. Neuer. 1993: In situ characterization of phytoplankton assemblages using fluorescence emission spectra. Mar. Biol., 115, 217-222.

____. R.J. Olson and S.W. Chisholm, 1988: Food selection by copepods: discrimination on the basis of food quality. Mar. Biol. 100, 4149.

Dagg, M., 1977: Some effects of patchy food environments on copepods. Limnol. Oceanogr. 22. 99-107.

Dam, H.G., C.A. Miller and S.H. Janasdottir, 1993: The trophic role of mesozooplankton at $47^{\circ}$ N, $20^{\circ} \mathrm{W}$ during the North Atlantic Bloom Experiment. Deep-Sea Res. II, 40, 197-212.

Denman. K.L. and A.E. Gargett, 1983: Time and space scales of vertical mixing and advection of phytoplankton in the upper ocean. Limnol. Oceanogr., 28, 801-815. and A.E. Gargett, 1988: Multiple thermoclines are barriers to vertical exchange in the subarctic Pacific during SUPER. May 1984. J. Mar. Res., 46, 77-103.

and T.M. Powell, 1984: Effects of physical processes on planktonic ecosystems in the coastal ocean. Oceanogr. Mar. Biol. Ann. Rev., 22, 125-168.

Desiderio, R.A.. T.J. Cowles, J.N. Moum and M.L. Myrick, 1993: Microstructure profiles of laser induced chlorophyll fluorescence spectra: evaluation of backscatter and forward scatter fiber optic sensors. J. Atmos. Ocean. Tech., 10, 209-224.

Donaghay, P.L., H.M. Rines and J.M. Sieburth, 1992: Simultaneous sampling of fine scale biological, chemical, and physical structure in stratified waters. Archiv: Hydrobiol. Beih., 36, 97-108.

Fasham, M.J.R., 1978: The statistical and mathematical analysis of plankton patchiness. Oceanogr. Mar. Biol. Ann. Rev., I6, 43 79.

Franks, P.J.S., 1995: Thin layers of phytoplankton: 
a model of formation by near-inertial wave shear. Deep-Sea Res., 42, 75-91.

Goldman. J.C., 1988: Spatial and temporal discontinuities of biological processes in pelagic surface waters. In: Toward a Theory of Biological and Physical Interactions in the World Ocean. B. Rothschild, ed. Kluwer. $273-296$.

Hanson. Jr.. A.K. and P.L. Donaghay. 1998: Microto fine-scale chemical gradients and layers in vertically stratified coastal waters. Oceanography. 11, 10-17.

Holliday, D.V., R.E. Pieper, C.F. Greenlaw and J.K. Dawson. 1998: Acoustical sensing of small scale vertical structure in zooplankton assemblages. Oceanography; 11, 18-23.

Kullenberg, G., 1974: Investigation of small-scale vertical mixing in relation to the temperature structure in stably stratified waters. Ads: Geophys., 18A, 339-351.

Lande, R. and M. Wood. 1987: Suspension times of particles in the upper ocean. Deep Sea Res.,

$$
34,61-72 .
$$

Lasker, R. 1975: Field criteria for survival of anchovy larvae: the relation between inshore chlorophyll maximum layers and successful first feeding. Fish. Bull, 73, 453-462.

Macintyre. S., A.L. Alldredge and C.C. Gotschalk, 1995: Accumulation of marine snow at density discontinuities in the water column Limnol. Oceanogr., 40, 449-468.

Mackas, D.L.. K.L. Denman and M.R. Abbott, 1985: Plankton patchiness: biology in the physical vernacular. Bull. Mar. Sci., 37, 652-674.

Marra, J., 1978: Effect of short-term variations in light intensity on photosynthesis of a marine phytoplankter: a laboratory simulation study. Mar. Biol., 46, 191-202.

Mitchell, J.G. and J.A. Fuhrman, 1989: Centimeter scale vertical heterogeneity in bacteria and chlorophyll a. Mar. Ecol. Prog. Ser., 54, 141-148.

Moum. J.N., D.R. Caldwell and C.A. Paulson. 1989: Mixing in the equatorial surface layer.
J. Geophys. Res., 94, 2005-2021.

Mullin, M.M. and E.R. Brooks, 1976: Some consequences of distributional heterogeneity of phytoplankton and zooplankton. Limnol. Oceanogr., 21, 784-796.

Oldham. C. and J. Imberger, 1992: Effects of hydrodynamics of oxygen transport paths in a lake Amer. Soc. Limnol. Oceanogr. 1992 Meeting, Sante Fe. New Mexico, February 1992.

Owen, R.W., 1989: Microscale and tinescale variations of small plankton in coastal and pelagic environments. J. Mar. Res., 47, 197240.

Rothschild, B.J. and T.R. Osborn, 1988: Small-scale turbulence and plankton contact rates. $J$. Plankton Res., 10, 465-474.

Tiselius, P., 1992: Behavior of Acartia tonsa in patchy food environments. Limnol. Oceanogr. 37 1640-1651.

Weller, R.A. and J.F. Price, 1988: Langmuir circulation within the oceanic mixed layer. $D^{\prime} e^{\prime} p$ Sea Res., 35, 711-747. 